\title{
PLC Impulsive Noise in Industrial Zone: Measurement and Characterization
}

\author{
Trung H. Tran, Dung D. Do, and Tue H. Huynh
}

\begin{abstract}
Noise in PLC is of relatively complex structure of which the most important component is the asynchronous impulsive noise. Based on measurements realized in an industrial zone, this paper shows the heavy tail phenomenon observed in experimental measures. Consequently, the alpha stable model is the most natural to be used in describing the statistics of PLC impulsive noise in industrial zones. Furthermore, the inter-arrival time is of Pareto distribution while the duration of impulses follows a mixed exponential distribution. All parameters of the alpha stable model as well as the Pareto parameters and the mixed exponents are statistically estimated. The variation of these parameters with time is also discussed.
\end{abstract}

Index Terms-Power line communication, impulsive noise, measurement and characterization.

\section{INTRODUCTION}

To design a good performance data transmission system that operates on the power line networks (PLC), it is necessary to know all impairments induced by the PLC networks used as communication channels. It is well known that PLC networks are complicated communication channels [1]. Due to many interconnections and taps, it is quite natural to model this kind of channels as random multipaths [2]-[4]. Furthermore, the mechanism generating additive noise is much more complex; based on experimental measurements as well as on physical modeling, additive noise on PLC is divided into different categories of which the most important component is the asynchronous impulsive noise that causes serious flaw to the data transmission systems that employ PLC as communication medium. The last few decades, many researchers have given many tries to model this noise component [5]-[7]. In our work, we are interested in measuring, analyzing and modeling this impulsive noise component in an industrial zone. Our measurements have shown that in such environment, the general characteristics are still observed (high amplitude, bursty) but in industrial zone, impulsive noise is of heavy tail. We organized a very intensive and complete campaign of measurement in order to obtain a very rich set of quite representative noise samples. Based on this experimental result, the heavy tail phenomenon

Manuscript received October 10, 2012; revised November 29, 2012.

Tran Huu Trung is with the Haiphong Private University, Vietnam (e-mail: trungth@hpu.edu.vn).

Do Duc Dung was with the Bacha International University, now with the Samsung Electronics Vietnam (e-mail: dddo@bhiu.edu.vn).

Huynh Huu Tue was with the Department of Electrical and Computer Engineering, Laval University, Canada, now with the School of Electrical Engineering, International University, HCM, Vietnam (e-mail: hhtue@hcmiu.edu.vn). is evident. Due to this conclusive observation, we propose to use the alpha stable distribution as model for impulse amplitude. Our approach is different from the known ones which often use Gaussian mixture to model this kind of noise [8]. In our work, we study the time varying characteristics of noise parameters, including amplitude statistic parameters, duration and inter-arrival time.

\section{MEASUREMENT}

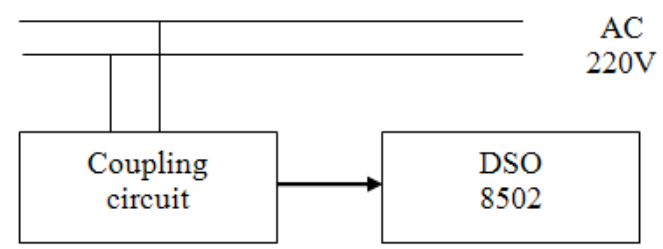

Fig. 1. Measurement set-up

We have conducted a measurement campaign in Do Son Industrial Park for 2 weeks, three times per day (i) from 8AM to $10 \mathrm{AM}$; (ii) from $11 \mathrm{AM}$ to $1 \mathrm{PM}$, and (iii) from $2 \mathrm{PM}$ to 4PM. Firstly, the electrical signal is extracted from the AC $220 \mathrm{~V} 50 \mathrm{~Hz}$ power line by an isolated coupling circuit and sampled at the rate of $500 \mathrm{MHz}$ by DSO8502, which can store $1,045,487$ samples in its 2MB RAM. To avoid recording too much data, the trigger level is set to $960 \mathrm{mV}$ and the recording time of each measurement is $524 \mu \mathrm{s}$. Results are then transferred to a PC and processed by a Matlab program.

\section{MEASUREMENT RESUlTS}

\section{A. Patterns of Measured Pulses}

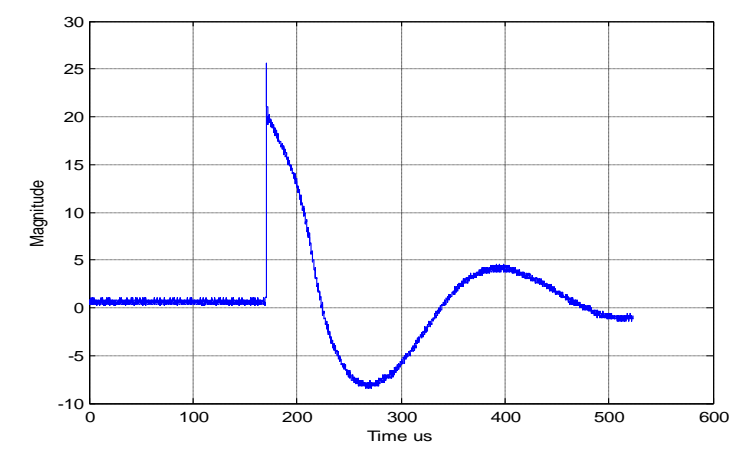

Fig. 2. A single decayed impulse

Fig. 2 shows a single decayed impulse. It has a vertical jumping at start and reduces by the exponentially-decaying-bound sinusoidal function. The pulse peak measured is up to $25 \mathrm{~V}$. We have observed 871 
pulses of this form during an hour and 48 minutes, which corresponds to a frequency of 0.1344 impulses/minute. The appearance of $25 \mathrm{~V}$-peak pulses at such high frequency can be seen as of heavy tail.

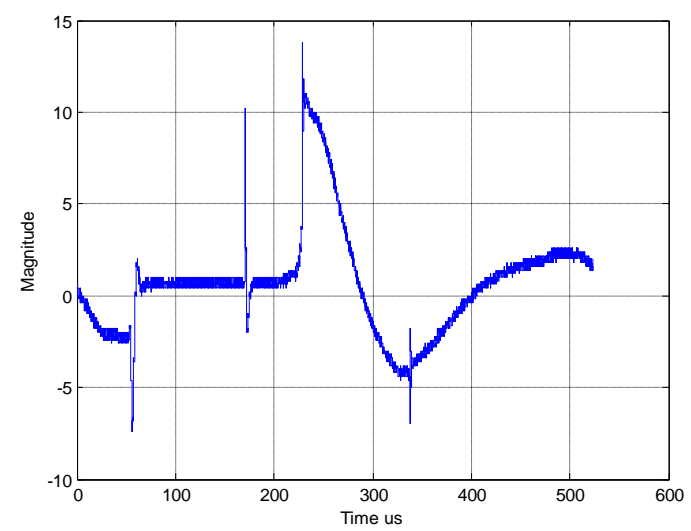

Fig. 3. Example of frequency of impulse

An observed sample (see in Fig. 3) shows us 3 impulses with interval time between each of them is rather small, from $100 \mu$ s to $200 \mu \mathrm{s}$. As the results of the measurement, we found that the industrial zone has affected strongly by impulsive noise with the high frequency of occurrence and the high magnitude. It can be considered as of heavy tail.

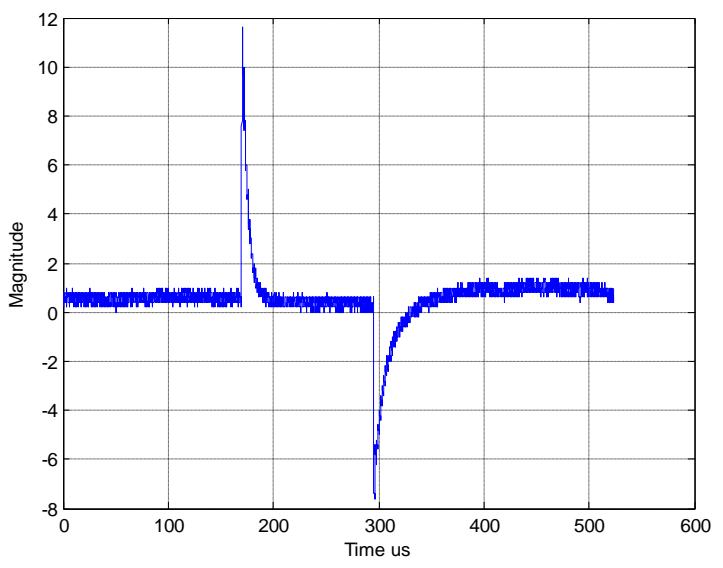

Fig. 4. Negative impulse

Fig. 3 shows a negative impulse. This impulse does not have the form of decreasing-bound impulse as described in [1], [4]. The existing of negative impulses requires us to study on $\mathrm{A}_{\text {imp- }}$ and $\mathrm{A}_{\text {imp+ }}$ seperately while building a magnitude distribution model.

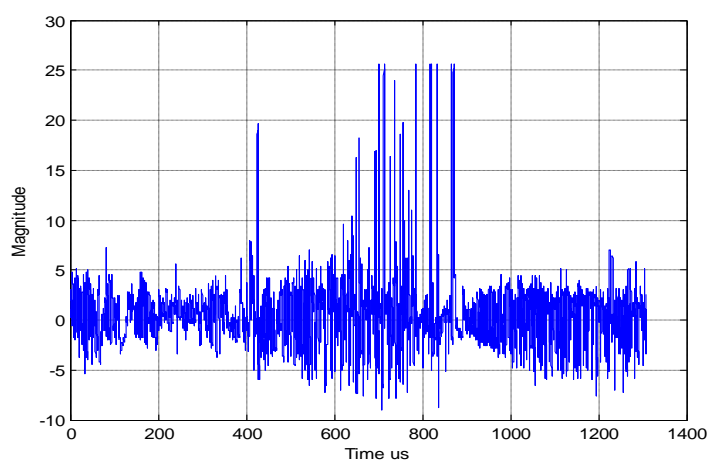

Fig. 5. Bursty impulsive noise

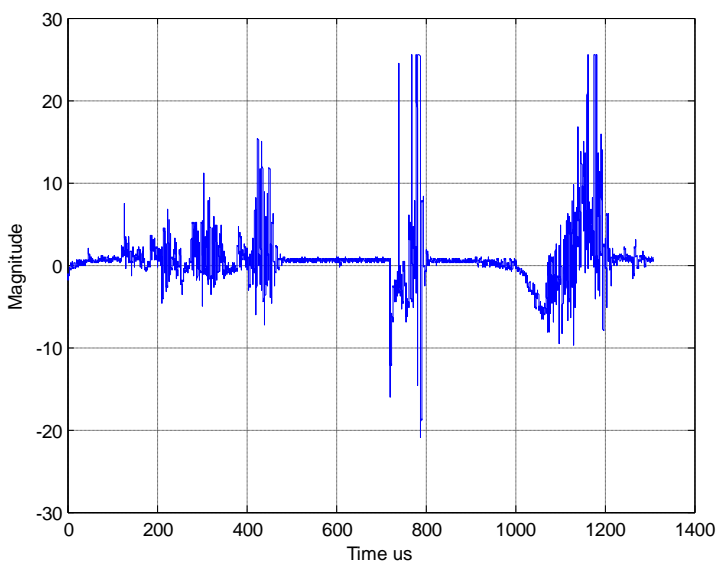

Fig. 6. Occurrence frequency of bursty impulsive noise

Fig. 4 and 5 show bursty impulsive noises whose form is not clearly determined[9], [10]. It composes of many single impulses and their magnitude can be high up to $25 \mathrm{~V}$. These high-magnitude impulses can seriously affect the performance of PLC systems.

\section{B. Magnitude of Impulse}

As previously discussed, given the heavy tail characteristic of impulsive noise in our measrement, we propse to use the alpha model [11] for industrial zone PLC impulsive noise. Characteristic function $\varphi(t)$ of alpha stable model is defined as follow:

$$
\varphi(t)= \begin{cases}\exp \left\{-\gamma^{\alpha} \cdot[t]^{\alpha} \cdot\left[1-i \beta \operatorname{sign}(t) \cdot \tan \frac{\pi \alpha}{2}\right]+i \delta t\right\} & \alpha \neq 1 \\ \exp \left\{-\gamma \cdot[t] \cdot\left[1+i \beta \frac{2}{\pi} \operatorname{sign}(t) \cdot \ln (t)\right]+i \delta t\right\} & \alpha=1\end{cases}
$$

where $\alpha \in(0,2] ; \beta \in[-1,1] ; \gamma \geq 0 ; \delta \in R$;

$$
\operatorname{sign}(t)=\left\{\begin{array}{cc}
1 & t>0 \\
0 & t=0 \\
-1 & t<0
\end{array}\right.
$$
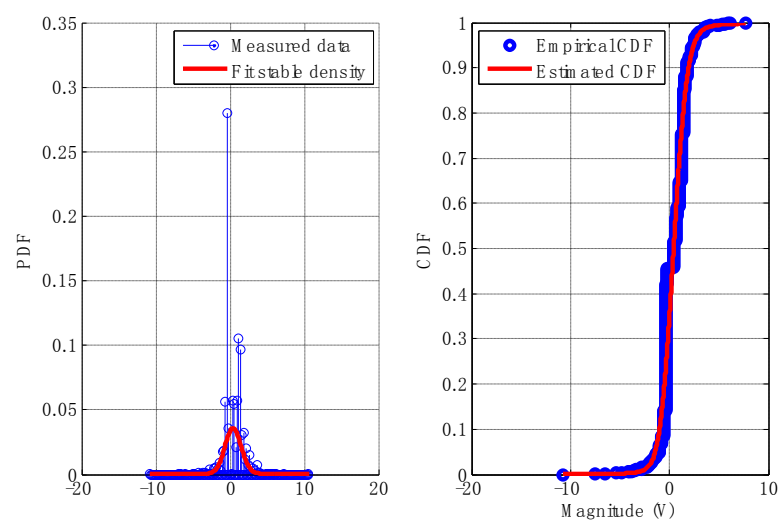

Fig. 7. PDF and CDF of magnitude of impulse measured from 11:00 to 13:00 at $29 / 7 / 2011$

Using the Maximum likelihood technique, we estimated the impulsive noise PDF function. The obtained values of the alpha stable distribution are $\alpha_{0}=1.8236, \quad \beta_{0}=0.3109$, $\gamma_{0}=0.78691, \delta_{0}=0.46598$. This distribution with $\beta_{0}>0$ expresses the deviation clearly tended to the positive 
direction.

TABLE I: THE FITTING RESUltS USING ALPHA DISTRIBUTION FOR DATA RECORDED DURING 29/JULY/2011 AT DiFFERENT TIME PERIODS.

\begin{tabular}{lllll}
\hline \hline Time & $\alpha_{0}$ & $\beta_{0}$ & $\gamma_{0}$ & $\delta_{0}$ \\
\hline 8:00 - 10:00 & 1.25924 & 0.758641 & 0.344402 & 0.675493 \\
11:00 - 13:00 & 1.8236 & 0.3109 & 0.78691 & 0.46598 \\
14:00 - 16:00 & 1.8284 & 1.42397 & 0.455938 & 0.45939 \\
\hline \hline
\end{tabular}

Table I shows the location parameter $\delta_{0}$ does not change much while the deviation $\beta_{0}$ change values according to different time periods. The slight change of parameters of $\alpha_{0}$, expresses that the distribution shape is not very sensitive to time frames.

The distribution of total magnitude at different time periods is shown in Fig. 8.
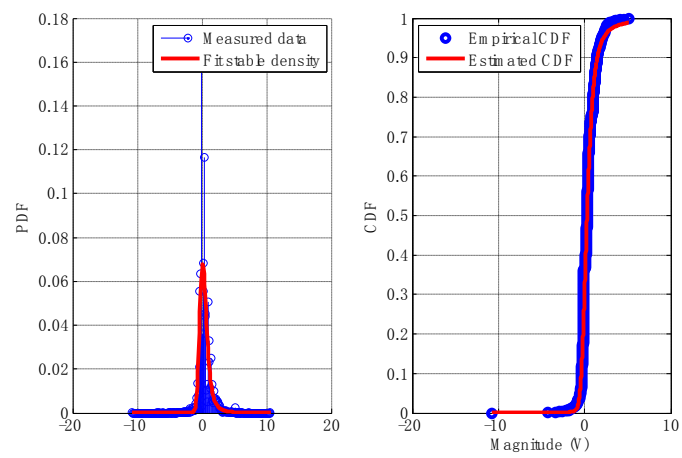

Fig. 8. Amplitude distribution for data obtained on the $29 / 7 / 2011$

TABLE II: THE FITTING RESULTS WITH DATA RECORDED IN DIFFERENT DAYS

\begin{tabular}{lllll}
\hline \hline Date & $\alpha_{0}$ & $\beta_{0}$ & $\gamma_{0}$ & $\delta_{0}$ \\
\hline $28 / 7$ & 1.11063 & 0.027578 & 0.265596 & 0.285798 \\
$29 / 7$ & 1.47117 & 0.895837 & 0.415185 & 0.583091 \\
$31 / 7$ & 0.853063 & 0.657667 & 0.113396 & -0.382411 \\
\hline \hline
\end{tabular}

\section{Duration of Impulse}

Analyzing the behavior of the impulse duration, it is clear that histogram of the impulse duration follow different exponential shape. To show that this type of mixed exponential distribution can be used with confidence, we have fitted many different situations of which the fitted result have shown that this model is a good choice. Fig. 9 shown a sample of the duration of impulse where we have proposed the sum of two exponential functions to fit the result.

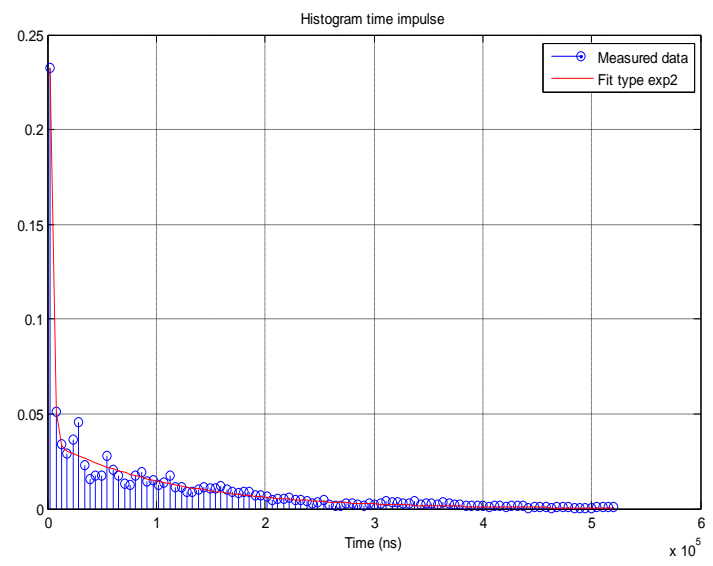

Fig. 9. Pdf of impulse duration
The parameters of the function $\mathrm{y}=\mathrm{a} \times \exp (\mathrm{b} \times \mathrm{t})+\mathrm{c} \times \exp (\mathrm{d} \times \mathrm{t})$ are $a=0.6601, b=-0.0004575, c=0.03579, d=-8.866 \mathrm{e}-006$.

\section{Inter-Arrival Time}
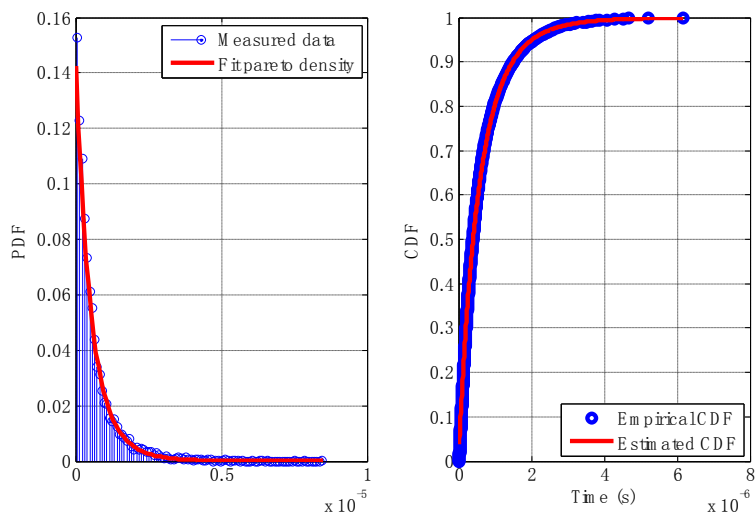

Fig. 10. PDF and CDF of inter-arrival time

The pdf of the inter-arrival time of the results measured from 8AM to 10AM, as shown in Figure 10, is appropriate to the Pareto distribution with $\mathrm{K}=0.099353$ và $\sigma=3.1652 \mathrm{e}-7$.

TABLE III: THE PARETO-FITTED RESUlTS OF INTER-ARRIVAL TIME OF THE DATA RECORDED DURING 3 DAYS $(28,29 \& 31 /$ JULY/2011).

\begin{tabular}{lll}
\hline \hline Date & $\mathrm{K}$ & $\Sigma$ \\
\hline $28 / 7$ (Working day) & 0.02112 & $3.9791 \mathrm{e}-7$ \\
29/7 (Working day) & -0.02759 & $3.8172 \mathrm{e}-7$ \\
$31 / 7$ (Sunday) & 0.1059 & $7.5649 \mathrm{e}-7$ \\
\hline \hline
\end{tabular}

\section{CONCLUSION}

We have organized a very intensive campaign of measurement of PLC asynchronous impulsive noise in an industrial zone. Through the experimental measurement results, we have observed the heavy tail phenomenon for the impulsive noise amplitude. For this observation, we proposed to use the alpha stable model for the amplitude distribution and the Pareto distribution for the inter-arrival time. We have also proposed a mixed exponential distribution for the impulse duration. When fitting with estimated parameters, the concordance between measured data and the theoretical curves is good

\section{REFERENCES}

[1] M. Zimmermann and K. Dostert, "An analysis of the broadband noise scenario in power-line networks," in proc. of International Symposium on Power-Line Communications and Its Applications, Limerick, pp. 131-138. , 5-7 April 2000.

[2] M. Zimmermann and K. Dostert, "A multi-path model for the power line channel," IEEE Transactions on Communications, vol. 50, no. 4, 2002, pp. 553-559.

[3] H. Philipps, "Development of a statistical model for power line communications channels," International Symposium on Power-Line Communications and Its Applications, Limerick, pp. 153-160. , 5-7 April 2000

[4] H. Chaouche, F. Gauthier, A. Zeddam, M. Tlich, and M. Machmoum, "Time domain modeling of powerline impulsive noise at its source," Journal of Electromagnetic Analysis and Applications, pp. 359-367. , March 2011

[5] V. Degardin, M. Lienard, P. Degauque, A. Zeddam, and F. Gauthier, "Impulsive noise on indoor power lines: characterization and mitigation of its effect on PLC systems," IEEE International Symposium on Electromagnetic Compatibility, Istanbul, vol. 1, pp. 166-169. 11-16 May 2003, 
[6] F. Kural and M. Safak, "An experimental investigation of impulse noise on low voltage powe rlines," International Symposium on Power-Line Communications and Applications, Athens, Greece, 27-29, March 2002.

[7] M. H. L Chan and R. W. Donaldson, "Amplitude, width and interarrival distributions for noise impulses on intrabuilding power line communication networks," IEEE Trans. Elec. Compatibility, vol. 3, no. 3, pp. 320-323, August 1989 .

[8] M. Nassar, K. Gulati, Y. Mortazavi, and B. L. Evans, "Statistical modeling of asynchronous impulsive noise in powerline communication networks," in Proc. of IEEE Global Communications Conference, Houston, USA., Dec. 5-9, 2011

[9] J. A. Weyepe Tina, A. J. Snyders, and H. C Ferreira, "Implementation of a gap recorder for measuring impulsive noise error distributions in power line communications using the fritchman model," International Symposium on PowerLine Communications and Applications, pp. 27-30, March 2012.

[10] P. Pagani, R. Hashmat, A. Schwager, D. Schneider, and W. Bäschlin, "European MIMO PLC field measurements: noise analysis," International Symposium on PowerLine Communications and Applications, 27-30, March 2012.

[11] G. Samorodnitsky and M. S. Taqqu, Stable Non-Gaussian Random Processes: Stochastic Models with Infinite Variance, Chapman and Hall, New York, 1994.

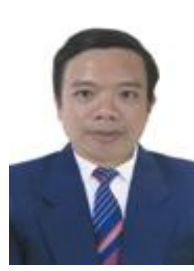

Tran Huu Trung was born in Haiphong, Vietnam in 1977. He received B.Sc. in Electronics Engineering from Haiphong Private University, Vietnam, in 2001 and M.Sc. degree from University of Engineering and Technology, Hanoi, Vietnam in 2005. From 2001 to 2007, he was an lecturer in the Department of Electronics, Haiphong Private University, Vietnam. His research interests include signal processing, powerline

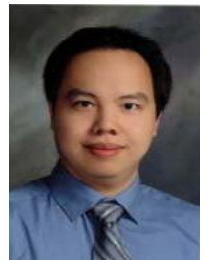

Dr. Do Duc Dung was born in Hanoi, Vietnam in 1979. He received M.Sc. and Ph.D. degree in information and computer technology from Chungbuk National University, Korea in 2004, and 2007, respectively. From 2008 to 2011, he was a lecturer of Bac-Ha International University, Vietnam. Since 2012, he has been a researcher in Samsung Electronics Vietnam. His current research interests include the signal processing, software solution.

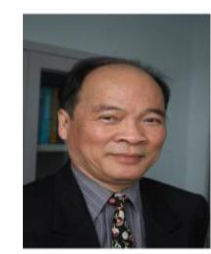

Prof. Huu Tue Huynh received the Sc.D. degree in 1972 from Laval University (Canada) where he had been a Faculty member of the Department of Electrical and Computer Engineering since 1969. He was an Invited Guest at The AT\&T Information Systems in Neptune, N.J. in 1984 and has been invited to give lectures at several Universities in Europe, America as well as in Asia. Professor Huynh is author and coauthor of two books and more than two hundred papers and reports in Information Processing. He has served as Consultant to a number of Canadian Government Agencies and Industries. His research interests cover stochastic simulation techniques, information processing, fast algorithms and architectures with applications to finance and to communications. In 2005, he left Laval University to create the Department of "Information Processing" at the College of Technology, VNU, Hanoi. During the period 2007-2011, he was invited to set up Bac-Ha International University, Hanoi, as her first President. Professor Huynh is now working as a research professor at the School of Electrical Engineering of VNU- HCM's International University, where his main responsibility is creating a new research group in "Intelligent Signal Processing". He is the Technical Editor-in-Chief of "REV-Journal on Electronics and Communication. 